\title{
ESTUDO DA SAZONALIDADE DE ALPHITOBIUS DIAPERINUS EM CRIAÇÃO DE GALINHAS POEDEIRAS POR TRÊS DIFERENTES MÉTODOS DE COLETA
}

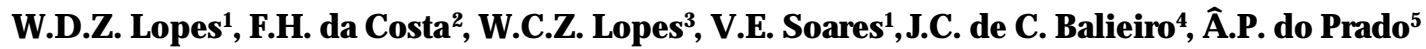

'Universidade Estadual Paulista, Faculdade de Ciências Agrárias e Veterinárias, Via de acesso Prof. Paulo Donatto Castellani, s/ no, CEP 14884-900, Jaboticabal, SP, Brasil. E-mail: wdzlopes@fcav.unesp.br

RESUMO

\begin{abstract}
A Iphitobius diaperinus(Panzer, 1979), consi deradouma praga secundáriadegrãosarmazenados, écomumenteencontrado comabundância nos substratos deaviários industriais em várias partes do mundo, causando prejuízos econômicos tanto direta quanto indiretamente. Foi realizado um levantamento deste tenebrionídeo em uma granja comercial de gal inhas poedeiras situada no Município de São João da Boa Vista, SP, entre janei ro de 2001 a dezembro de 2002, utilizando-se trêsmétodosdistintos decol eta: armadilhas desolo, funil deBerlese-Tull gren eflutuação emágua. Do total deinsetos coletados, armadil has de solo somaram $94,19 \%$ detoda população, 1,78\% pelo funil deBerlese-Tull gren e4,01\% de cascudinhos capturados pel a flutuação emágua. Esta espécie decoleóptero ocorreu nas 75 coletas realizadasnos 24 mesesdeexperimento, sendo as armadilhas de solo o método mais sensível $(P<0,05)$ para captura de $A$. diaperinus.
\end{abstract}

PALAVRAS-CHAVE: A vespoedeiras, esterco deaves poedeiras, produção animal, Tenebrionidae.

\section{ABSTRACT}

STUDY OF THE SAZONALITY OF ALPHITOBIUS DIAPERINUS IN CREATION OF LAYER HOUSE FORTHREE DIFFERENT METHODSOF COLLECTION. A Iphitobius diaperinus, considered asecondary plague of stored grains, is commonly found in ind ustrial aviaries excrementsin some parts of the world. It causes economic damages in poultry houses in such a way directly, as well as indirectly. A survey of thistenebrioni daein acommercial grangesituated in SãoJ oão daBoaVista city was carried out from January 2001 to December 2002, by using three distinct collect methods: soil trap, funnel of Berlese-Tullgren and fluctuation in water. Of thetotal of insects, $94.19 \%$ were collected by soil trap, $1.78 \%$ by funnel of Berlese-Tullgren and $4.01 \%$ by fluctuation in water. This coleopteran wasfound in all the 75 collectscarried outduring the 24 monthsof experiment, and the soil trap the most sensible method $(P<0.05)$ for the capture of $A$. diaperinus.

KEY WORDS: Layer house, lagged layer manure, Tenebrionidae, animal production.

INTRODUÇÃO

O cascudinho, A Iphitobius diaperinus (Panzer), é um besouro da família Tenebrionidae, originário do Oeste A fricano, considerado uma praga secundária degrãosarmazenadosecomumenteencontrado com abundâncianosaviáriosindustriaisemvárias partes do mundo, ondesecriaem gal pões defrango decorte, de postura, em gal pões de matrizes e perus (PEFFEIFER \& AXTELL, 1980).

A expansão da avicultura industrial em sistemas de confinamento nas últimas décadas propiciou a este tenebrionídeo um habitat ideal para seu desenvolvimento, tornando o "cascudinho" um problema mundial.

Em criações intensivas de perus, frangos de corte e de postura, este besouro pode se tornar um sério problema, proliferando-se em grandes quantidades nacama, naração eno acúmulo deestercoondelarvas e adultos se desenvolvem.

Estecoleóptero vem sendo registrado como reservatório de uma grande variedade de patógenos de grande importância para a avicultura (HoFSTAD et al ., 1972). Entreosmicrorganismosisolados, encontram-

${ }^{2}$ M édico Veterinário, Autônomo, São J oão da Boa Vista, SP, Brasil.

3Universidade de São Paulo, Faculdade de M edicina, Ribeirão Preto, SP, Brasil.

${ }^{4}$ Universidade de São Paulo, Faculdade de Zootecnia e Engenharia Alimentar, Pirassununga, SP, Brasil.

5UNICAMP, Instituto de Biologia, Departamento de Parasitologia, Campinas, SP, Brasil. 
se fungos como o Fusarium sp., A spergillus sp. eCândida sp. (De Las Casas et al., 1968; De Las Casas et al., 1973; De LASCASAset al ., 1976; A Lveset al ., 2004). Foram isolados 48 sorotipos de Escherichia coli (McAlisteret al., 1996), Salmonella thyphimurium, Salmonella sp., Bacillus sp. Enterobactérias e Streptococcus sp. (EIDsoN et al., 1965; Eidson et al., 1966; Lefreret al., 2002). Dentre os vírus, foram isolados dos besouros os causadores do Gumboro, Leucose, DoençadeM areck, N ew castle eRotavírus (Edison et al., 1966; De LASCASAset al ., 1973; Fenner et al., 1987). A Coccidiose, principal doença causada pelo protozoário Eimeria sp., sobrevive no acúmulo de fezes das aves, porém os oocistos deste coccídeo podem ser ingeridos pel os besouros eassim serem consumidos regularmente pelas aves (REYNA et al., 1983; A puYA et al., 1994).

A transmissão destespatógenosocorrequandoas aves ingerem larvas e adultos de A. diaperinus infectados. Durante os dez primeiros dias de vida, tanto frango de corte quanto aves em postura podem ingerir cerca de 450 larvas/ ave/ dia, podendo apresentar fezes líquidas com conteúdo aparentemente não digerido (TSENG et al., 1971).

A lém dos prejuízosacima citados, os besouros da famíliaTenebrionidae, ao serem molestados, liberam uma secreção defensi va para se livrarem dos predadores. No caso doA . diaperinus, isolou-sedessasecreção as quinonas que são substâncias tóxicas e carcinogênicas, que podem levar a lesões hepáticas podendo determinar a condenação desse órgão no abatedouro (TSENG et al., 1971). Outra problemática ocasionada por este besouro é a danificação nos sistemas de isolamento térmico de galpões climatizados, quando perfuram o material isolante, 0 que prejudica seriamente o desenvolvimento inicial dasaves, determinando resultadosinsatisfatóriosno ganho depeso ou na produção deovos (TURNER, 1986).

NoBrasil, poucostrabal hos básicostêm sidofeitos na área, dificultando assim, um maior entendimento do que ocorre com esta espécie neste ecossistema artificial. Os mais importantes são: A AGESEN (1988), BRUNo et al. (1993), Fernandes et al. (1995), Bicho et al. (2005) e Pinto et al. (2005). Dentre estes, Pinto et al. (2005) se destacado ponto devistaecológico, jáqueestesautores estudaram(emgranjadeavespoedeirasdePel otas, RS) a flutuação populacional deste tenebrionídae, capturados por armadilhas do tipo sanduíche.

Deste modo, como este coleóptero pode causar grandes prejuízos econômicos em granja avícola, associado à defasagem do número de trabal hos realizadosnaárea, éessencial determinar asazonalidade desta espécie dentro das estações do ano de 2001 e 2002, comparando-se qual método de coleta que se mostra mais sensível para captura desteartrópode, a fim de auxiliar o controle desta praga, por meios de métodos e manejo integrado.

\section{MATERIAL E MÉTODOS}

\section{D escrição da G ranja}

As coletas foram realizadas na granja Crisdan, situadaa $7 \mathrm{~km}$ do MunicípiodeSãoJ oão daBoaVista, Estado deSão Paulo (22으' S, 046048' W, altitudede $763 \mathrm{~m})$.

A granja, considerada de pequeno porte, possuía 2 conjuntos de 4 gal pões (abertos) cada, com capacidade para al ojar 25.000 gal inhas, das linhagens "Hy Line" e "Hy-line Brown", em fase de postura.

O gal pão decoleta possuía 3 conjuntos degaiolas (ou 3 corredores ondeseacumulam as fezes) separados por 2 corredores de concreto de 0,5 m delargura. As filei ras das gaiolas estão distribuídas em degrau (tipo"narrow house"). A sgaiolasvariam detamanho, podendo abrigar de 2 a 4 gal inhas. Sob as gaiolas, o piso é de terra onde as fezes se acumulam.

A oredordagranjahaviaculturaagrícola,eduranteo período analisado, a predominância foi de cafée milho. Durante a coleta, nenhum inseticida foi utilizado sobreo esterco, o qual foi retirado em 24/ 4e20/ 9 de 2001 eem 15/ 3e 2/ 11 de 2002. Devido as al tas preci pitaçõespluviométricas constatadasno verão, 0 uso deóxido decálcioedeserragemfoi relativamente comum.

Foram realizadas setenta ecinco coletas entre17/ 1/ 2001 a 26/ 12/ 2002, com interval os de 7 dias durante os primeiros 12 meses, passando a quinzenais nos demais meses, totalizando 1.125 armadilhas e $600 \mathrm{~kg}$ de esterco. Para amostragem dos espécimes adultos foram utilizados 3 métodos distintos; armadilha-de-solo (método 1), o funil deBerlese-Tullgren (método 2) ea flutuação em água (método 3).

\section{M étodo 1 - A rmadilha-de-solo}

Estas armadil has foram utilizadas visando a obtenção de tenebrionídeos adultos (WALKER, 1985; SUNMERLIN, 1989). Consistiu na utilização de frascos plásticos com $9,0 \mathrm{~cm}$ de altura e $8,0 \mathrm{~cm}$ de diâmetro contendo $200 \mathrm{~mL}$ deumlíquidoconservanteefixador (80\% deágua; $5 \%$ deglicerina; $5 \%$ deálcool a $70 \%$; $5 \%$ deformol e $5 \%$ de detergente) para posterior identificação equantificação em mi croscópio esterioscópio. As armadilhas foram retiradas e substituídas por outras com novo fixador em cada coleta, sendo elas numeradas de 1 a 15, as quais foram escolhidas al eatoriamente e posteriormente enterradas sob as gaiolas, ao longo de3corredores(cinco/ armadilhas/ corredor) A, B eC, próximo ao esterco acumulado.

A distribuição das armadilhas foi feita por "amostragemsistemática", queconsistiu em del imitar áreasem quadradoscontíguos $\left(1 \mathrm{~m}^{2}\right)$, ondeelasforam distribuídas (KREBS, 1989). 


\section{M étodo 2 - Extração direta do esterco}

O esterco acumulado sob as gai ol as apresentava, visual mente, 5 tipos distintos de consistência: líquido, pastoso, pastoso-firme, firme e seco (Bruno et al., 1993), sendo queno gal pão haviaesterco com umaou mais consistências diferentes. As amostras foram retiradas de forma a obter esterco detodas as consistências.

$\mathrm{Na}$ coleta das amostras de esterco foi utilizada uma pá, ao invés da introdução de um cilindro de metal, como recomendado por PECK \&A NDERSON (1970). Para obtenção deste material, foram extraídas cerca de 10 ( $400 \mathrm{~g} /$ amosta) amostras al eatórias num total de aproximadamente $4 \mathrm{~kg}$ dos diferentes tipos de esterco por coleta.

Em seguida, o material coletado foi transferido parafunil deBerlese-Tullgren confeccionado comum tubo de papel cartolina sobre um vidro, com líquido fixador econservador (álcool a 70\%). Sobreo funil foi utilizada uma lata ti po embalagem de achocolatado sem fundo quefoi substituída por uma tela demal ha de $1 \mathrm{~cm}$. O esterco (cerca de $670 \mathrm{~g} /$ funil) era colocado dentro da lata e ficava exposto a lâmpada de $25 \mathrm{~W}$, durante 5 dias.

\section{M étodo 3: Flutuação em água}

As amostras foram extraídas do esterco utilizando-se o método de Robets recomendado por MoOrE (1954), com base no princípio de flutuação.

Da mesma forma que o método anterior, cerca de 10 amostras ( $400 \mathrm{~g} /$ amostra) foram extraídas al eatoriamente do galpão para obtenção do material, totalizando aproximadamente $4 \mathrm{~kg}$ de esterco por coleta.

Em8baldesde10L decapacidade, contendoágua quase até a borda, foram colocadas porções de cerca de $500 \mathrm{~g}$ deesterco/ balde. A pós agitação feita com o auxíli o deum bastão demadeira eposterior decantação do esterco, foram coletadas os artrópodes que flutuavam, com o auxílio de uma peneira de malha fina. Os espécimes obtidos foram secados em papel toal ha, para posterior identificação.

Todo material coletado foi levado para o laboratório onde foi triado, contado, identificado, fixado e conservado em álcool a $70 \%$, sendo que para a identificação do "cascudinho" foram utilizados os trabaIhos de Chu (1949), Wenzel (1955) e Borror \& White (1970). O material testemunho encontra-se na Coleção Entomológica da Facul dade de M edicina Veterinária do Centro Universitário das Faculdades Integradas da Fundação de Ensino "Octávio Bastos".

Osdadosdesazonalidadereferentesaos 3 métodos de col eta ao longo dos 2 anos de coleta foram previamentetransformadosem $\log (x+1)$ e, posteriormente, efetuou-se a análise de variância por meio de um delineamento em parcela subdividida no tempo ("Split Plot in Time"), sendo estabeleci dos como tratamento principal os métodos de coleta e como tratamento secundário, as estações dos anos para obtenção das significâncias do teste $F$. As médias $(=\Sigma[\log (x+1)] / n)$ dostratamentosforamobtidaspelo procedimento Lsmeans utilizando o Teste TukeyKramer $(P<0,05)$.

As análises das correlações momento-produto dePearson foram utilizadas para aval iação de quão associadas foram os números de A . diaperinus capturados etemperatura (ambiente), umidaderelativa do ar e os índices pluviométricos. Os dados pluviométricos, de temperatura e umidade relativa do ar, foram fornecidos pelo Banco de Dados Hidrometerológicos da Cooperativa Agrícola Nacional Sudeste Centro Oeste (COPERSERVE), situada em São J oão da Boa Vista, SP. O teste para verificação de correlações realizada pelo programa supracitado é um Testet de Student.

Para tanto, foram utilizados os procedimentos GLM do programaStatistical A nalysis System, versão 6.12 .

\section{RESULTADOSE DISCUSSÃO}

Coletaram-se 57.767 tenebrionídeos adultos pertencentes à espécieA I phitobius diaperinus, sendo que, onúmerodecoleópterosdiferiu quando secomparam os 3 métodos de captura. $N$ as coletas real izadas por meio dearmadilhas desolo foramencontrados 52.532 besouros $(94,19 \%)$ do número total, utilizando-se a flutuação em água foram encontrados 2.240 (4,01\%) e, pelo funil de Berlese-Tullgren, foram coletados apenas 995 (1,78\%) tenebrionídeos de toda população ao longo dos 24 meses de coleta.

No presente estudo em granja deaves poedeiras, a armadil ha de solo mostrou ser o método mais sensível $(P<0,05)$ para captura de $A$. diaperinus em relação ao funil de Berlese-Tullgren e à flutuação em água (Tabela 1). Além disso, neste método de coleta, não foram encontradas diferenças estatística $(P>$ $0,05)$ no número de $A$. diaperinus capturados nas diferentes estações dos anos (primavera, verão, outono einverno de2001e2002) aolongo do experimento. Entretanto paraflutuação emáguaeofunil deBerleseTullgren, verificou-seum baixo número significativo $(P<0,05)$ detenebrionídeos na primavera em relação ao outonoeinvernodosanosde2001e2002(Tabela1).

As correlações entre os totais de A. diaperinus encontradosedadosclimáticos (temperaturaumidaderelativadoar eprecipitação pluvial) foram debaixa magnitude, indicando baixas associações com estas covariáveis (Tabela 1). 
Tabela 1 - Resultado das comparações multiplas (médias ajustadas - Tukey-Kramer ) do desdobramento da interação estações do ano (2001 e2002) e métodos de captura, em granja aviária de São João da Boa Vista, SP.

\begin{tabular}{lcccr}
\hline Métodos de col eta & \multicolumn{2}{c}{ Estações dos anos/ Médias $=S[\log (x+1) / n]$ de A l phitobius diaperinus } \\
\cline { 2 - 5 } & Primavera & Verão & Outono & Inverno \\
\hline armadilhas desolo & $2,7285 \mathrm{~A}$ & $2,8640 \mathrm{~A}$ & $2,6633 \mathrm{~A}$ & $2,6935 \mathrm{~A}$ \\
& $\mathrm{a}$ & $\mathrm{a}$ & $\mathrm{a}$ & $\mathrm{a}$ \\
flutuação em água & $0,4315 \mathrm{~B}$ & $0,6946 \mathrm{~B}$ & $1,3431 \mathrm{~B}$ & $1,0536 \mathrm{~B}$ \\
& $\mathrm{C}$ & $\mathrm{abc}$ & $\mathrm{a}$ & $\mathrm{ab}$ \\
funil deBerlese-Tullgren & $0,3063 \mathrm{~B}$ & $0,8388 \mathrm{~B}$ & $0,8837 \mathrm{~B}$ & $0,8809 \mathrm{~B}$ \\
& $\mathrm{~b}$ & $\mathrm{ab}$ & $\mathrm{a}$ & $\mathrm{a}$ \\
\hline
\end{tabular}

Coeficiente de correlação das covariáveis Temperatura (TEMP), U midade relativa (UR) e Pluviometria (PP) para A . diaperinus capturados em armadilhas desolo, flutuação em água efunil de Berlese-Tul lgren.

\begin{tabular}{llll}
\hline & Temp $(\stackrel{\circ}{ } \mathrm{C})$ & UR $(\%)$ & PP $\left(\mathrm{mm}^{3}\right)$ \\
A lphitobius diaperinus & $0,07448 \mathrm{~ns}$ & $-0,16858 \mathrm{~ns}$ & $0,15009 \mathrm{~ns}$ \\
\hline
\end{tabular}

*Médias seguidas por pelo menos uma letra em comum, minúscula na linha emaiúscula na coluna não diferem entre si pelo TesteTukey $(\mathrm{P}>0,05)$

${ }^{*}=$ Significativo $(P \varangle 0,05)$

${ }^{*}=$ Significativo $(P<0,01)$

${ }^{n s}=$ Significativo $(P>0,05)$

Tabel a 2 - Total deA I phitobius diaperinus nas diferentes estações do ano, capturad os segundo o método deamostragem, em granjas de aves poedeiras de São João da Boa Vista,SP.

\begin{tabular}{lccrc}
\hline $\begin{array}{l}\text { Estaç̃̃es dos anos } \\
\text { (2001e 2002) }\end{array}$ & Armadilhas desolo & funil deBerlese-Tullgren & flutuação emágua & Total \\
\hline Primavera & & & & \\
Verão & $13.275,00$ & 48,00 & 63,00 & $13.386,00$ \\
Outono & $12.927,00$ & 374,00 & 133,00 & $13.434,00$ \\
Inverno & $14.811,00$ & 329,00 & $1.168,00$ & $16.308,00$ \\
\hline Total & $11.519,00$ & 244,00 & 876,00 & $12.639,00$ \\
\hline
\end{tabular}

Tabela 3 - Resultado das comparações multiplas (médias ajustadas - Tukey-Kramer ) do desdobramento da interação corredor e número da armadil ha de captura, em granja aviária de São J oão da Boa Vista, SP.

\begin{tabular}{llll}
\hline Nodaarmadilhadesolo & Corredor A & Corredor B & Corredor C \\
\hline A rmadilha1 & $37,034 \mathrm{~A}$ & $33,2780 \mathrm{~A}$ & $34,3120 \mathrm{~A}$ \\
A rmadilha2 & $33,998 \mathrm{~A}$ & $34,9350 \mathrm{~A}$ & $35,6090 \mathrm{~A}$ \\
A rmadilha3 & $34,093 \mathrm{~A}$ & $38,9910 \mathrm{~A}$ & $31,9760 \mathrm{~A}$ \\
A rmadilha4 & $34,181 \mathrm{~A}$ & $35,8610 \mathrm{~A}$ & $36,9700 \mathrm{~A}$ \\
A rmadilha5 & $36,085 \mathrm{~A}$ & $34,5790 \mathrm{~A}$ & $34,7070 \mathrm{~A}$ \\
\hline Médias =S[log $(\mathrm{x}+1) / \mathrm{n}]$ & $35.078 \mathrm{a}$ & $35.529 \mathrm{a}$ & $34.715 \mathrm{a}$ \\
\hline
\end{tabular}

*M édias seguidas por pelo menos uma letra em comum, minúscula na linha emaiúscula na col una não diferem entresi pelo TesteTukey $(\mathrm{P}>0,05)$.

A temperatura durante o período de coleta apre sentou mínima de 160 C (junho de 2001) emáxima de 34으 (janeiro eoutubro de2001), umidaderelativa do ar apresentou mínima de $24 \%$ (agosto de 2001) e máxima de $100 \%$ (maio de 2001), para pluviosidade foi notificadamínima de $0 \mathrm{~mm}^{3}$ nos meses dejulho de 2001 e 2002 e máxima de $341 \mathrm{~mm}^{3}$ em novembro de 2001 (Figs. 1, 2e 3). 


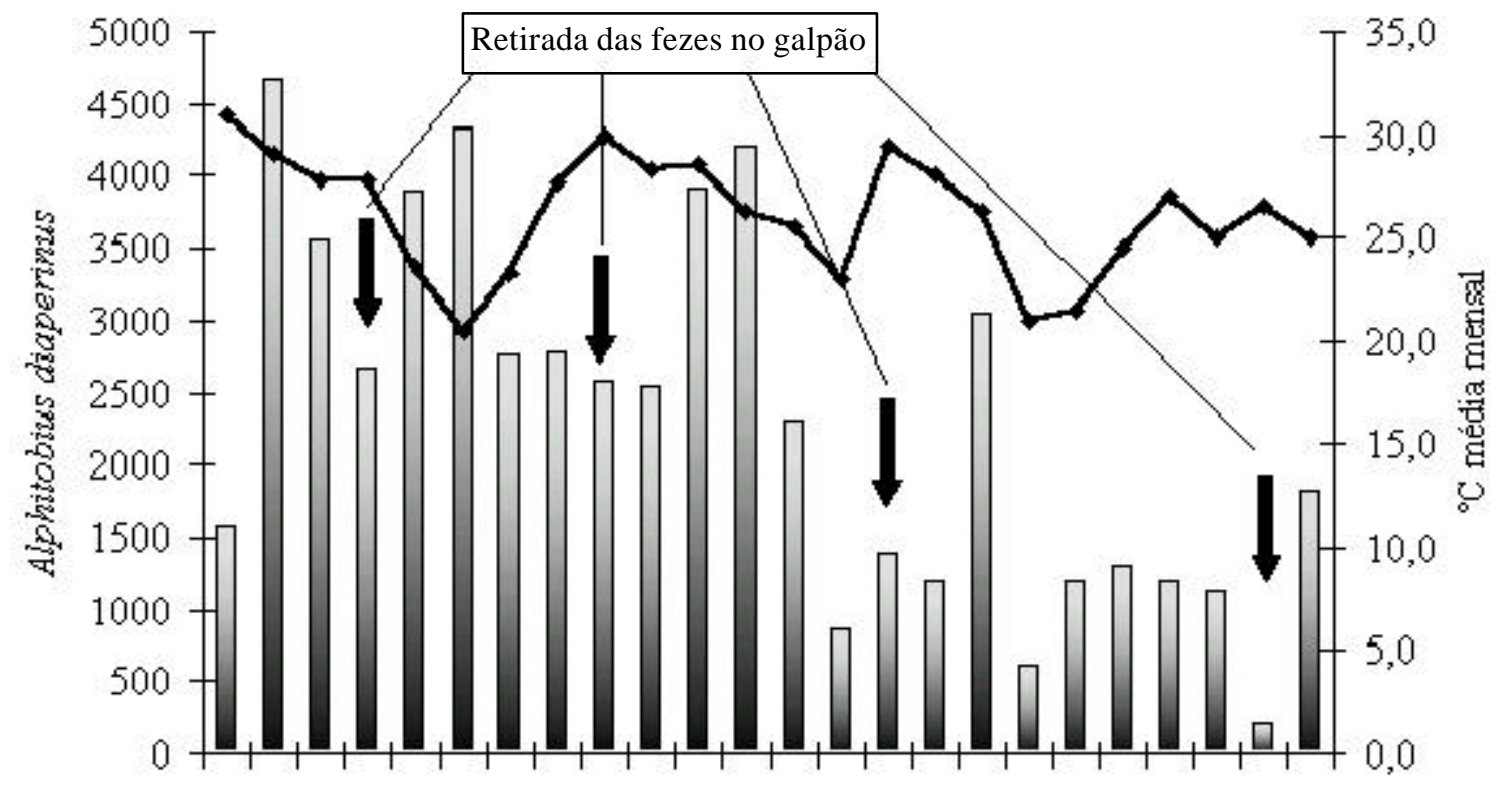

J F M A J J A S O N D J F M A J J A S O N D

Meses

\section{Alphitobius diaperinus $\rightarrow$ Temperatura ${ }^{\circ} \mathrm{C}$}

Fig. 1- Total deA I phitobius diaperinus capturadosem armadilhas desolo, flutuação emágua efunil deBerlese-Tullgren, edados sobrea temperatura média mensal dejaneiro de 2001 a dezembro de 2002, em granja avícola de São João da Boa Vista, SP.

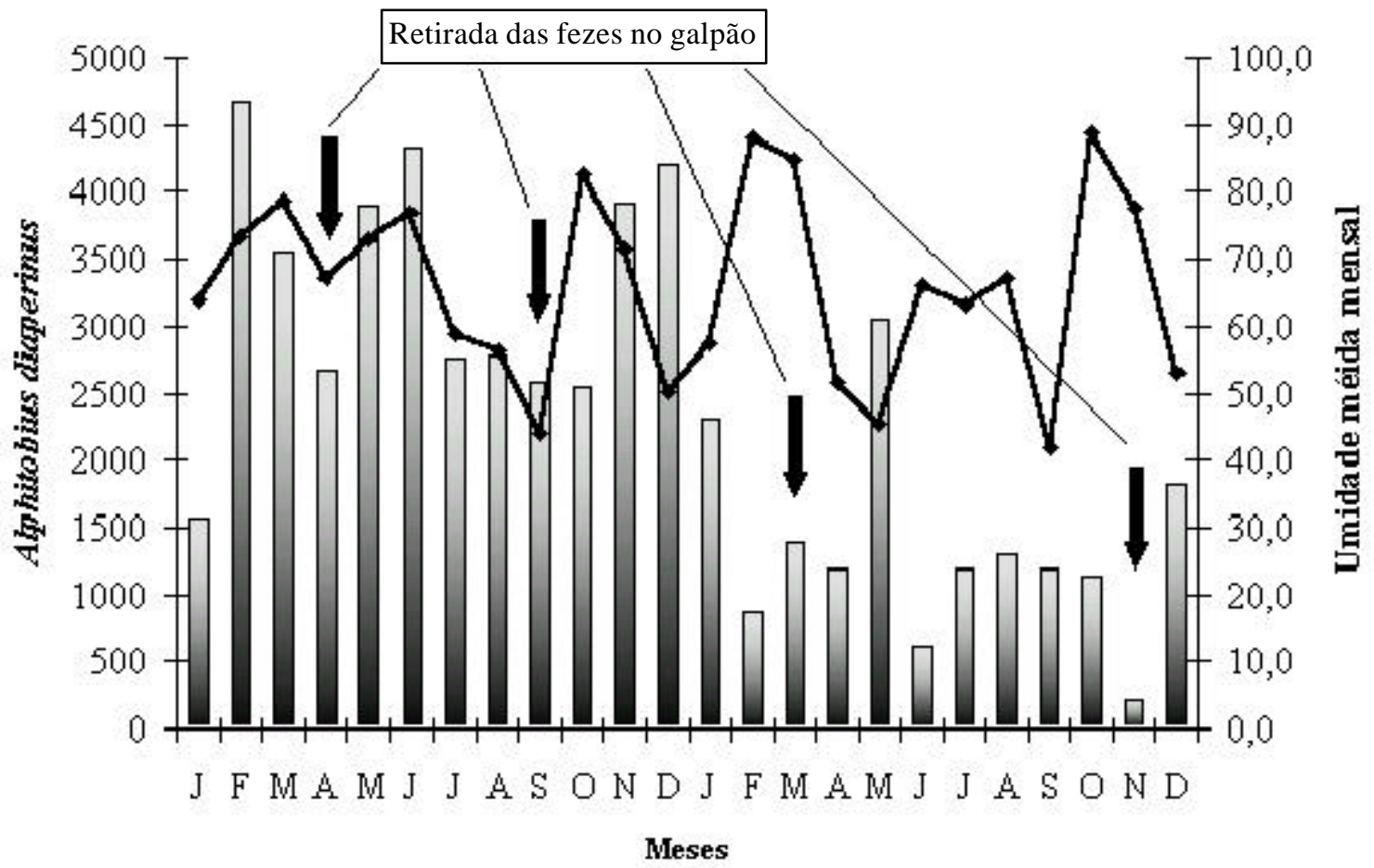

Elphitobius diaperinus $\rightarrow$ Umidade relativa $(\%)$

Fig. 2-Total deA I phitobius diaperinus capturadosem armadilhas desolo, flutuação emágua efunil deBerlese-Tul lgren, edados sobrea umidade (média) relativa do ar mensal dejaneiro de 2001 a dezembro de2002, em granja avícola deSão João da Boa Vista, SP. 


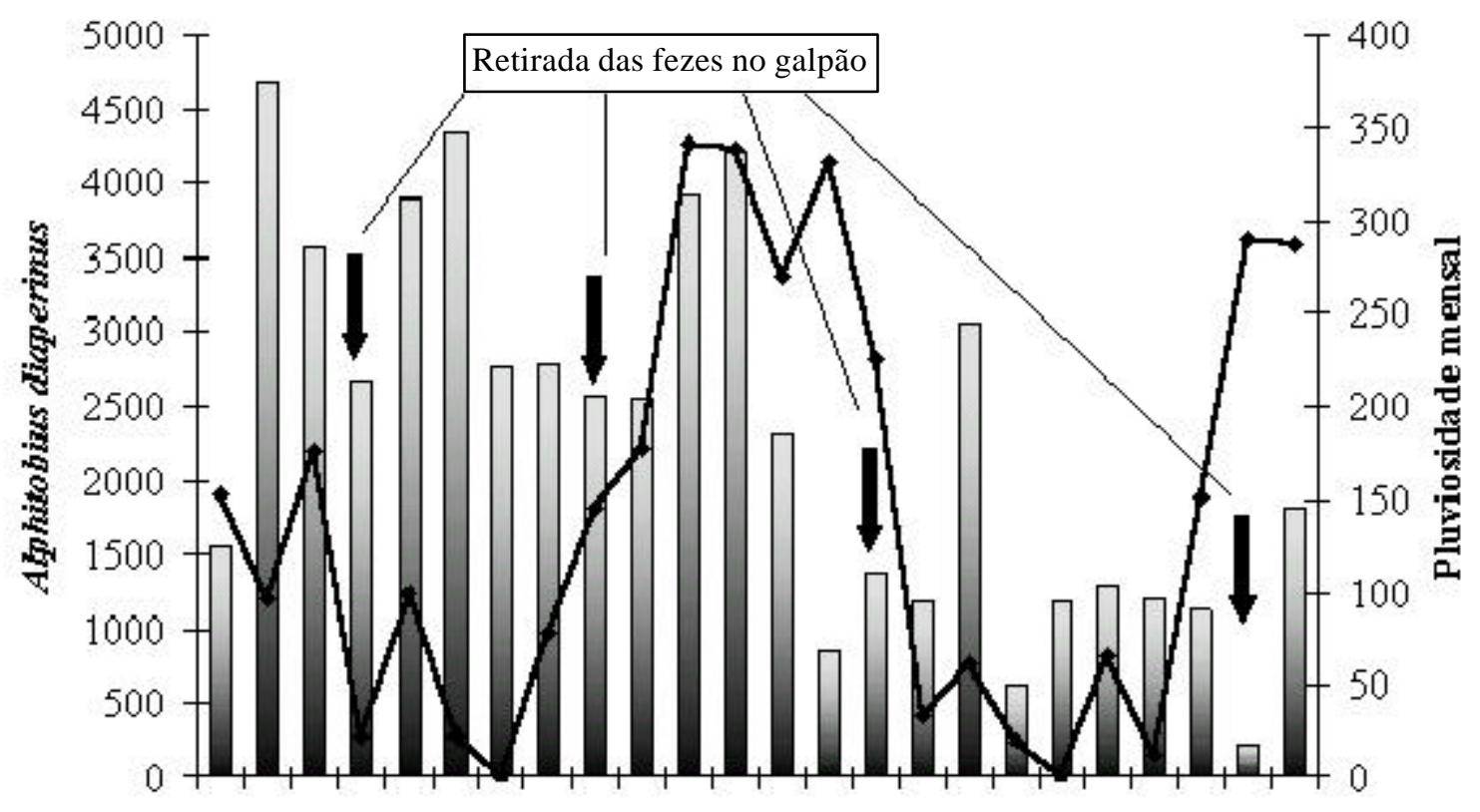

J F M A J J A S O N D J F M A J J A S O N D

Meses

\section{$\Longrightarrow$ Alphitobius diaperinus $\rightarrow$ Pluviosidade (mm3)}

Fig. 3-Total deA Iphitobius diaperinus capturados em armadilhas desolo, flutuação em água efunil de Berlese-Tullgren, edad os sobrea precipitação pluviométrica mensal dejaneiro de2001 a dezembro de2002, em granjaavícola deSãoj oão da Boa Vista, SP.

O maior índice de captura de A. diaperinus foi registrado em fevereiro de 2001 (4.679) com temperatura média de $29^{\circ} \mathrm{C}$ (Fig. 1). A menor ocorrência foi assinalada em novembro de 2002 com 221 tenebrionídeos capturados, sendo queo baixo número deA . diaperinus coletadosnestemêscoincidiu com a retirada de fezes do gal pão de coleta (Fig. 1).

Estudando a flutuação populacional de A. diaperinus capturados por armadilhas doti posanduíche, em Pelotas, RS, PInto et al. (2005) verificaram maior índice de captura (adultos) no mês de março (11.721), quando a temperatura média mensal foi de $22,20^{\circ} \mathrm{C}$, a menor ocorrência deu-se no mês de julho (25). Resultados semel hantes foram encontrados por BıcHo et al. (2005) também na mesma granja (Centro Agrotécnico Visconde de Graça, CAVG).

Nas estações do ano do presente estudo, o acme populacional de A . diaperinus (coletados pelos 3 métodos de captura) foi no outono de 2001 e 2002, com $16.308(28,23 \%)$ coleópteros detodapopulação levantada, entretanto, no inverno de2001 e2002, foi encontrado o menor pico populacional de tenebrionídeos (12.639 ou 22,66\%) (Tabela 2).

Francisco (1996), em esterco deaves poedeiras em granjas do Município deM onteM or, SP, verificou que adultos elarvas deA . diaperinus são mais numerosos nos meses mais frios do ano (outono e inverno) e sensivelmente reduzidas nos meses mais quentes (primavera everão), quando a precipitação das chuvas foi maior, desfavorecendo o desenvolvimento de larvas e adultos. Despins et al. (1989) relatam que o aumento daumidade do esterco provoca o abandono dassuasformasimaturas, indo estasparalocaismais secos como a estrutura do aviário.

A . diaperinus (tenebrionidae) também foi assinalado por Aagesen (1988), Bruno et al. (1993), Fernandes et al . (1995), Bichoet al . (2005) ePINToet al . (2005). Bicho et al. (2005), ao correlacionarem diversas espécies de besourosem granjaavícola dePelotas, RS, mencionaramestecoleópterocomosendoosegundomaisabundante (2.896). FernANDEs et al. (1995), em Uberlândia, MG, relataram A . diaperinus como a mais abundante na respectiva granja, perfazendo 56,89\% dos 3.011 coleópteros capturados. Bruno et al . (1993), em diversas granjas de vários municípios do Estado de São Paulo, registraram A diaperinus como o terceiro coleóptero mais abundante nos aviários em questão. Sua presença foi notificada por LEGNER\& OLTON (1971) em excrementos de galinhas, de bovinos edesuínos, e por LomÔnaco \& Prado (1994), Bcho et al. (2005) e 
Pinto et al. (2005) em excrementos de aves poedeiras em Uberlândia, MG e Pelotas, RS.

Convém salientar que não houve diferenças estatísticas $(P<0,05)$ no total de $A$. diaperinus capturados nosdiferentes corredores ( $\mathrm{A}, \mathrm{BeC}$ ) em queasarmadiIhas desoloestiveram dispostas (cinco/ armadilhas/ corredor) aleatoriamente, ou seja, o número de "cascudinhos" nagranja Crisdan foi homogêneo por todo gal pão decoleta utilizando-seesta metodologia (Tabela 3).

É importante ressaltar que, de um modo geral, ocorramal terações no número deartrópodescapturados em relação aos meses do ano de tal forma que, quando se aproxima dos meses de temperaturas amenas, a populaçãodecoleópterostendeadiminuir, uma vez quedependedeal ta temperatura eumidade para se desenvolver (Merchant et al., 1985; Hulley, 1986; Costa,1989 e Mendes \& LinHARES, 2002).

Entretanto, Pinto-Coelho (2000) descorreu que, além dos fatores climáticos (temperatura, umidade relativa do ar e precipitação pluviométrica), outros podem interagir na sazonalidade dos artrópodes, como osdependentes dadensidade (disponibilidade de alimento, espaço, predação) e os fatores independentes da densidade (componentes genéticos, interação social e dispersão).

\section{CONCLUSÕES}

Combasenosdadosencontrados, pode-seinferirque: A sazonalidadedeA . diaperinus podeou não tornarse homogênea nas diferentes estações do ano, dependendo do método deamostragem adotado $(P<0,05)$.

$\mathrm{N}$ otificou-seumaquedasignificativa $(\mathrm{P}<0,05)$ do numero total deA . diaperinuscoletados pelaflutuação em água e o funil de Berlese-Tullgren na primavera, em relação ao outono e inverno de 2001 e 2002.

Em granja deaves poedeiras, armadilhas desolo mostraram-semaissensível $(P<0,05)$ paracapturade A . diaperinus em relação ao funil deBerlese-Tullgren eflutuação em água.

O estudo populacional de A. diaperinus "cascudinho", associadosaesterco deavespoedeiras, deveser feito por mais deum método decoleta, como descrito neste estudo.

REFERÊNCIAS

AAGESEN, T.L.A rtrópodes associadosà excrementosem aviários. 1988. 37p. Dissertação (Mestrado)-EscolaSuperior de Agricultura Luiz de Queiroz, Universidade de São Paulo, Piracicaba, 1988.

Alves, L.F.A.; Alves, V.S.; Bressan, D.F.; Neves, P.M.O.J.; Alves, S.B. Ocorrência de $M$ etarhizium anisopliae (Metsch.) Sorok. emadultosdecascudinho (A I phitobius diaperinus) (Panzer) (Coleoptera:Tenebrionidae) em aviários comerciais em Cascavel, PR. N eotropical Entomology, v.33, p.793-795, 2004.

Apuya, L.C.; Stringram, S.M.; Aredens, J.J.; Broocks, W.M. Prevalenceof protozoan infectionsindarkling beetles from poultry houses in North Carolina. Journal of Invertebrate Pathology, v.63, p.255-259, 1994.

Bicho, C.L.; Almeida, L.M.; Ribeiro, P.B.; Silveira Junior, P. Flutuação populacional circanual decoleópteros em granja avícola em Pelotas, RS, Brasil. Iheringia, Série Zoologia, v.95, p.205-212, 2005.

BorRoR, J.D.\&W HITE, E.R.Insects. Boston:Houghton Mifflin Company, 1970. 404p.

Bruno, T.V.; Gumarães, A.M.M.; Tucci, E.C. Moscas sinantrópicas (Diptera) eseus predadores quesecriam em esterco de aves poedeiras confinadas, no Estado de São Paulo, Brasil. Revista Brasileira de Entomologia, v.37, p.577-590, 1993.

Chu. H.F. The immature insects. Iowa: Brown Company Publishers/ lowaWesleyan College, 1949. 234p.

Costa, V.A. Parasitóides pupais (H ymenoptera: Chal cidoidea) deM usca domestica L. 1758, Stomoxys cal citrans (L. 1758) e M uscina stabulans (Fallen, 1816) (Diptera: M uscidae) em aviárias de Echoporã, SP. 1989. 53p. Dissertação (Mestrado) - EscolaSuperior deA gricultura “Luiz de Queiroz", UniversidadedeSãoPaulo, Piracicaba, 1989.

De Las Casas, E.; Pomeroy, B.S.; Haren, P.K. Infection and quantitative recovery of Salmonella typhimurium and Echerichia coli form within the lesser Mealworm (Alphitobius diaperinus). P oultry Science, v.47, p.18711875, 1968.

De LasCasas, E.; H arein, P.K.;D eshmuck, D.R.; Pomeroy,B.S. The relationship between the lesser mealworm and avian viruses. I. Reovirus 24. Environmental Entomology, v.2, p.1043-1047, 1973.

De LasCasas, E.; H arien, P.K.; Deshmukh, D.R.; Pomeroy,B.S. Relastionship beween thelesser M ealw orm, fowl pox and Newcastle disease virus in poultry. Journal of Economic Entomology, v.69, p.775-779, 1976.

Despins, J.L.; Turner, Junior, E.C.; Ruszler, P.R. Effects of poultry manure moisture an poultry house construction materialsnothemovmentsof thelesser mealworm, Alphitobius diaperinus (Panzer) (Coleoptera: Tenebrionidae), a structural insect pest in high rise caged layer houses. Poultry Science, v.68, p.1326-1331, 1989.

Eidson , C.S.; Schimittle, S.C.; LaL, J.B.; Goode, R.B. Therole of darkling beetle, Alphitobius diaperinus in transmission of acute leucosis in chickens. Poultry Science, v.44, p.1366-1367, 1965.

EIdSON ,C.S.;SchimitTle, S.C.; LAL, J.B.; Goode, R.B. Induction of leucosis tumors with the beetle Alphitobius diaperinus. A merican J ournal of $V$ eterinary R eserch, v.27, n.119 p.1053-1057, 1966.

Fenner,F.;Bachmann,P.A.;G ibs, E.P.;M URPhy,F.A.;Studdert, M.J.; WHITE, D.O. V eterinary virology. San Diego, CA: Academic Press, 1987. 327p.

Fernandes, M.A.;Santos, M.A.; Lomônaco, C. Ocorrênciade artrópodes no esterco acumulado em uma granja de galinhas poedeiras. Anais da Sociedade Brasileira de Entomologia, v.24, p.649-653, 1995. 
Francisco, O. Alphitobius diaperinus (Panzer) (Coleoptera: Tenebrionidae) associado a esterco em granjas de aves poedeiras: fenologia, estrutura etária e parasitismo. 1996. 116p. Dissertação (Mestrado) - Instituto de Biologia, UniversidadeEstadual deCampinas, Campinas, 1996.

Hofstad, M.; Calneck, B.W.; Helmbolt, C.F.; Redid, W.V.; Yoder, H.W.D iseases of poultry. 7.ed.A mes: IowaStates Univ. Press, 1972. 234p.

H ULLEY, P.E. Factors affecting numbers of $M$ usca domestica L. (Diptera: M uscidae) and someother files breeding in poultry manure. Journal of Entomology Social South A frica, v.49, p.19-27, 1986.

KreBs, C.J. Ecological methods. New York: Haper \& Row, 1989. xii +700p.

Legner E.F.\& OTON, G.S. Distribuition and relative abundanceof dipterouspupaeand their parasitoidsin accumulations of domestic animal manure in Southwestern United States. H ilgardia, v.40, p.81-100, 1971.

Lefter, C.; Biesdorf, S.M.;A lmeidA, L.M.;Lefter, E.V.B.;VIGne, F. Isolamento de Enterobactérias em Alphitobius diaperinus enacamadeaviários noOestedo Estado do Paraná, Brasil . R evista Brasileira de Ciência A vícola, v.4, p.234-247, 2002.

LomônAco, C. \& PRADO, A.P. Estrutura comunitária e dinânima populacional da fauna de dípteros e seus inimigos naturais em granja avícolas. A nais daSociedade Brasileira de Entomologia, v.23, p.71-80, 1994.

McAlister, J.C.; Stellman, C.D.; Skeeles, J.K.; Newberry, L.A.; GBUR, E.E. Reservior competence of Alphitobius diaperinus (Coleoptera: Tenebrionidae) forE scherichia coli. Journal of M edical Entomology, v.31, p.369-372,1996.

MENDESJ. \& LinhARES, A.X. Cattledung breeding dípterain pasteur in southeastern brazil: diversity, abundance and seasonality. M emórias do Instituto O swaldo Cruz, v.97, p.37-41, 2002.

Merchant, M.E.; Flanders, R.V.; Willians, R.E. Sampling methodscomparisonsfor estimation of parasitism of M usca domestica (Diptera: Muscidae) pupae in accumulated poultry manure. Journal of Economic Entomology, v.78, p.1299-1303, 1985.
Moore, I. An efficient method of collecting dung beetles. Panamericam Pacific Entomology, v.30, p.208-216, 1954.

PECK, R.F. \& ANDERSON , J.R. Influence of poultry manure removal schedules on various diptera larval and selected arthropod predators. Journal of Economic Entomology, v.63, p.82-90, 1970.

Pefeifer, R.W. \&AxtelL, R.C.Coleopteraof poultry manure Incages-layer housesin N orth California. Envronmental Entomology, v.9, p.21-28, 1980.

Pinto-Coelho, R.M. Fundamentos deecologia. Porto Alegre. Artmed, 2000. 247p.

PInto,D.M.;R IBEIRo, P.B.; Bernard, E. Flutuação populacional deA I phitobius diaperinus (Panzer, 1979) (Coleóptera: Tenebrionidae), capturados por armadilhas do tipo sanduíche, em granja avícola, no município dePelotas, RS. Arquivos do Instituto Biológico, São Paulo, v.72, p.199-203, 2005. Disponível em: 丸ttp:/ / www. biologico.sp.gov.br/ arquivos/ V72 1/ pinto.pdf $>$. Acesso em 19 set. 2005.

Reyna, P.S.; McDadougald, L.R.; Mathis, G.F. Surviral of cocida in poutry litter and reservoirs of infevtion. A vian Disease, v.27, p.464-473, 1983.

SUMmERLIN, J.W. Tetchiness for collecting, rearing and handling histerid beetles. Southwestern E ntomologist, v.14, p.415-425, 1989.

Tseng, Y.L.; Davidson , J.A.; Menzer, R.E. Morphology and chemistry of the odorifroos gland of the lesser mealworm, A. diaperinus (Coleoptera: Tenebrionidae). A nnals Entomol ogy of Society A merican , v.64, p.425-430, 1971.

TURnER, E.C. Structural and litter pests.P oultry Science, v.65, p.425-430, 1986.

WALKER, R.L. A pitffal trap study on the Carabidae and Staphylinidae (coleoptera) in Country Durham. Entomology M onth M anagement, v.121, p.1-18, 1985.

WENZEL, R.L. Thehisterid beetleof N ew Caledonia.A nnual Review Ecology System, v.37, p.601-638, 1955.

Recebido em 3/ 4/ 06 A ceito em 7/ 6/ 06 